\section{GW23-e2491 INEQUALITIES IN LIFESTYLE BEHAVIOURS, DIETARY HABITS AND FAMILIAL FACTORS AMONG NORMAL WEIGHT, OVERWEIGHT OR OBESE CHINESE CHILDREN AND ADOLESCENTS}

doi:10.1136/heartjnl-2012-302920d.21
${ }^{1}$ Xiaofan Guo, ${ }^{2}$ Liqiang Zheng, ${ }^{1}$ Yang Li, ${ }^{1}$ Shasha Yu, ${ }^{1}$ Hongmei Yang, ${ }^{1}$ Xinghu Zhou, ${ }^{1}$ Xingang Zhang, ${ }^{3}$ Zhaoging Sun, ${ }^{1}$ Yingxian Sun. ${ }^{1}$ Department of Cardiology, the First Affiliated Hospital of China Medical University, Shenyang; ${ }^{2}$ Department of clinical epidemiology, Library, Shengjing Hospital of China Medical University, Shenyang; ${ }^{3}$ Department of Cardiology, Shengjing Hospital of China Medical University, Shenyang

Objectives Paediatric obesity has become a global public health problem. Data on the lifestyle behaviours, dietary habits and familial factors of overweight and obese children and adolescents are limited. The present study aimed to compare the health related factors among normal weight, overweight and obese Chinese children and adolescents.

Methods We conducted a cross-sectional study consisted of 4262 children and adolescents aged 5-18 years. Anthropometric measurements and information on health related variables, such as physical activities, sleep duration, dietary habits, family income and recognitions of weight status from views of both children and parents, were collected by well-trained personnel.

Results The prevalence of overweight and obesity was $15.3 \%$ and $6.4 \%$, respectively. Compared to girls, boys had a much higher prevalence of either overweight $(17.5 \%$ vs $12.9 \%)$ or obesity $(9.5 \%$ vs $3.1 \%$ ). About half of the parents with an overweight or obese child failed to recognise their child's abnormal weight status, and $65 \%$ with an overweight child would not take measures to decrease their child's body weight. Obese children and adolescents had higher likelihood to be nonsnackers (OR: 1.348, 95\% CI 1.039 to 1.748 ) or to have a family income $2000 \mathrm{CNY}$ or more per month (OR:1.442, 95\% CI 1.045 to 1.99$)$ and were less likely to have longer sleep ( $>=7.5 \mathrm{~h}$ ) (OR:0.475, 95\% CI 0.31 to 0.728 ) compared to the ones with normal weight.

Conclusions Our study indicated a high prevalence of paediatric overweight and obesity in a large Chinese population. Inequalities in sleep duration, snacking, family income and parental recognitions of children' weight status among participants in different weight categories were observed, which should be taken into account when planning prevention and treatment programmes of paediatric obesity. 